University of Nebraska - Lincoln

DigitalCommons@University of Nebraska - Lincoln

P. F. (Paul Frazer) Williams Publications

Electrical \& Computer Engineering, Department

August 1992

\title{
An optical technique for measurement of semiconductor surface electric fields
}

Harshad P. Sardesai

The University of Texas at Arlington, Applied Physical Electronics Research Center, Arlington, Texas

William C. Nunnally

The University of Texas at Arlington, Applied Physical Electronics Research Center, Arlington, Texas

Follow this and additional works at: https://digitalcommons.unl.edu/elecengwilliams

Part of the Electrical and Computer Engineering Commons

Sardesai, Harshad P. and Nunnally, William C., "An optical technique for measurement of semiconductor surface electric fields" (1992). P. F. (Paul Frazer) Williams Publications. 41.

https://digitalcommons.unl.edu/elecengwilliams/41

This Article is brought to you for free and open access by the Electrical \& Computer Engineering, Department of at DigitalCommons@University of Nebraska - Lincoln. It has been accepted for inclusion in P. F. (Paul Frazer) Williams Publications by an authorized administrator of DigitalCommons@University of Nebraska - Lincoln. 


\title{
An optical technique for measurement of semiconductor surface electric fields
}

\author{
Harshad P. Sardesai and William C. Nunnally \\ The University of Texas at Arlington, Applied Physical Electronics Research Center, Arlington, \\ Texas 76019-0308 \\ P. F. Williams \\ University of Nebraska-Lincoln, Department of Electrical Engineering, Lincoln, Nebraska 68588.051I
}

(Received 24 January 1992; accepted for publication 28 April 1992)

\begin{abstract}
We present an optical technique for the measurement of semiconductor surface electric fields. The measurement technique uses the Kerr electro-optic effect in nitrobenzene, a phase sensitive interferometer, and associated data acquisition units to measure the surface electric fields between the contacts of a planar semiconductor device. This technique was used to measure the surface fields on silicon devices used in pulsed power applications, but has the potential for use as an electric field probe for any device having high surface electric fields, both pulsed and dc. The measurement technique showed a temporal resolution of $100 \mathrm{~ns}$, which can be easily reduced to a few nanoseconds using superior data acquisition and detection systems. The spatial resolution was about $50 \mu \mathrm{m}$ for devices that had a typical contact separation of about $500 \mu \mathrm{m}$ (power devices). This technique can be applied to measure the surface fields on devices commonly used in microelectronic applications. In this paper we discuss in detail the measurement technique and present the results obtained for silicon photoconductive power switches.
\end{abstract}

\section{INTRODUCTION}

Semiconductor devices used for pulsed power as well as microelectronic applications generally have electrodes placed in geometry's where high electric fields are developed across their surface. An understanding of the surface electric fields is very important especially for characterizing the breakdown properties of the device. Surface breakdown normally occurs at a lower threshold than bulk breakdown. Further, the semiconductor surface is complicated due to the presence of surface states, surface contamination, surface irregularities and surface heating. All these factors interplay to make an analytical solution of the surface electric fields very complex. Hence experimental techniques are a very attractive method to determine these surface fields in real time.

Optical techniques are best suited for this purpose as they are fast, noninvasive, and have considerably less error. Optical techniques based on the Kerr effect have been used for measuring high-voltage pulses. ${ }^{1,2}$ Surface field probing techniques based on the electro-optic Pockel effect ${ }^{3}$ and the Franz-Keldysh effect ${ }^{4}$ have been reported in the literature. We use the Kerr electro-optic effect and a phase sensitive interferometer to measure the surface fields. This technique produces a finite fringe interferogram (an interferogram with background fringes), which is indicative of the electric fields. The sample whose surface fields are to be measured is a silicon photoconductive power switch, which basically consists of a slab of very pure, nearly intrinsic silicon with aluminum ohmic contacts. The sample is mounted inside the Kerr cell and a pulsed high voltage is applied to it. A linearly polarized, cw, He-Ne laser beam traverses the region along the width of the contacts, and undergoes a phase change proportional to the square of the electric field present. The phase is analyzed with an optical interferometer which interacts the two polarization components to form interference fringes. The change in phase is determined by using a streak camera to streak the image from a slit that is parallel to the semiconductor surface. The streak image on the film is of the form of a continuous flow image which gives the temporal portion of the measurement. The spatial portion is obtained by noting the change in individual fringes as the spatial extent of the fringes on the film corresponds to the gap between the contacts. Advantages of this technique include the presence of background fringes which facilitates the accurate determination of partial fringe shifts and makes it possible to distinguish between increasing and decreasing field values. Further, a continuous observation of the fields during the voltage application is possible. The limitations on this technique are imposed by the quality of the optical system used and on the resulting fringe visibility and readability.

The authors had previously reported some preliminary results ${ }^{5}$ of this measurement technique which were based on two different arrangements of the interferometric analyzer. These results were very encouraging, and hence one of the analyzer arrangement was pursued further mainly because the theory behind it was well established and the empirical constants required by the theory were well documented. This approach was, however, not successful in accurately determining the surface fields, ${ }^{6}$ and had to be eventually discarded. The other analyzer arrangement was then used and the theory behind its operation was developed. This arrangement required the knowledge of the electro-optic coefficient of nitrobenzene (which serves as the electro-optic medium) which were detcrmined by calibrating the Kerr cell. For a more complete description of 


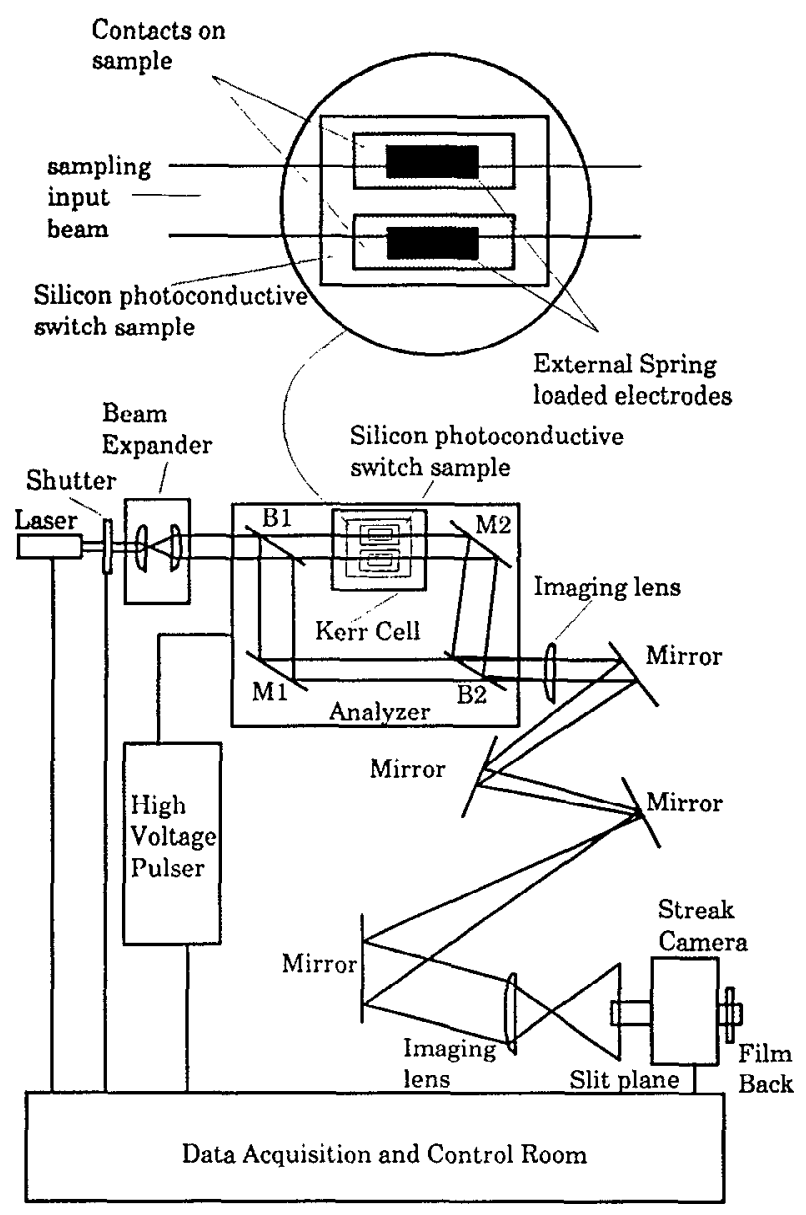

FIG. 1. Schematic diagram of experimental arrangement.

the experimental arrangement and the theory the reader is referred to Refs. 5 and 7.

\section{EXPERIMENTAL ARRANGEMENT}

Figure 1 shows the block diagram of the experimental arrangement. Plane polarized light from a $20 \mathrm{~mW} \mathrm{He}-\mathrm{Ne}$ laser with the plane of polarization at $45^{\circ}$ in the $x-y$ plane is passed through the beam expansion optics. The expanded beam is split by the input beam splitter B1 located in the analyzer section. Horizontally polarized light gets transmitted and is made to pass through the Kerr cell where it samples the entire area between the contacts of the photoswitch sample kept inside the Kerr cell. It is then directed by mirror M2 onto the output beam splitter B2. The reflected vertically polarized light which is the reference beam is passed through a half-wave plate which rotates the polarization to make it horizontal and mirror M1 directs it onto output beam splitter B2. At the output of beam splitter $\mathrm{B} 2$ the two polarization states are made to interfere to produce a sharp fringe image of the sample surface. This fringe image is made incident on the slit of the streak camera with the slit placed just above the fringe image of the sample surface. The sampling beam is shuttered for $5 \mathrm{~ms}$ by an external mechanical shutter to prevent damage to the streak tube and improve the final image on the film. The voltage pulse is applied by a high-voltage pulser which delivers microsecond flat-topped voltage pulses across the switch contacts. The entire experiment is controlled through the data acquisition and control room from where the various triggering delays are adjusted and the sample voltage and current are monitored.

Briefly the experiment is operated as follows. Initially, the various optical pieces are carefully adjusted and the mechanical shutter is then triggered to open, allowing the laser beam to pass through and form the background fringes across the slit of the streak camera. A trigger is then given to the streak camera to initiate the streak recording and after a certain delay, the high-voltage pulser is triggered to apply a $800 \mathrm{~ns}$ high-voltage $(0-5 \mathrm{kV})$ pulse across the switch contacts. The phase of the sampling beam is changed by the integral of the electric field present between the contacts. ${ }^{8}$ This change in phase causes the fringes to shift in time and as the streak recording was started before the application of the high-voltage pulse (i.e., when the electric field was zero). The final fringe image on the film is of the form a continuous flow image of the fringes, with the fringes appearing bent during the time period that the voltage pulse was applied. The output light intensity is given $b y^{7}$

$$
I \propto \cos ^{2} \frac{\gamma-\Delta_{E}}{2},
$$

where $\gamma$ is the background phase difference (without electric field) and $\Delta_{E}$ is the electric field induced phase retardation and is given by ${ }^{7}$

$$
\Delta_{E}=2 \pi \frac{\Delta y}{\delta y},
$$

where $\delta y$ is the background fringe spacing and $\Delta y$ is the induced fringe shift. The fringe shift is measured and the electric field in space and time is calculated by using the relation ${ }^{7}$

$$
\Delta_{E}=-\frac{\pi l}{\lambda} n^{3} s_{11} \mathbf{E}(x, y, t)^{2},
$$

where $l$ is the length of the birefringent path, $\lambda$ is the free-space wavelength, $n$ is the isotropic refractive index, $s_{11}$ is the electro-optic coefficient of nitrobenzene, and $\mathbf{E}(x, y, t)$ is the surface electric field.

\section{KERR CELL CONSTRUCTION AND CALIBRATION}

The Kerr cell is perhaps the most important component of the experiment and was custom designed and machined and is shown in Fig. 2. It is made from aluminum with outer dimensions of $4.8 \mathrm{in} . \times 4.8 \mathrm{in} . \times 4.8 \mathrm{in}$. The sample is placed on the bottom of the Kerr cell on a round glass pedestal, and two optical windows, $1 \mathrm{in}$. in diameter on two opposite faces of the cell allow the laser beam to pass through the cell. The windows are high-quality optical glass flats and are fitted in a special housing to reduce strain and maintain a good degree of parallelism. A 2.5in.-diam viewport allows for direct observation of the semiconductor surface. The bottom surface of the cell consists of yet another glass window to provide for contact illumi- 

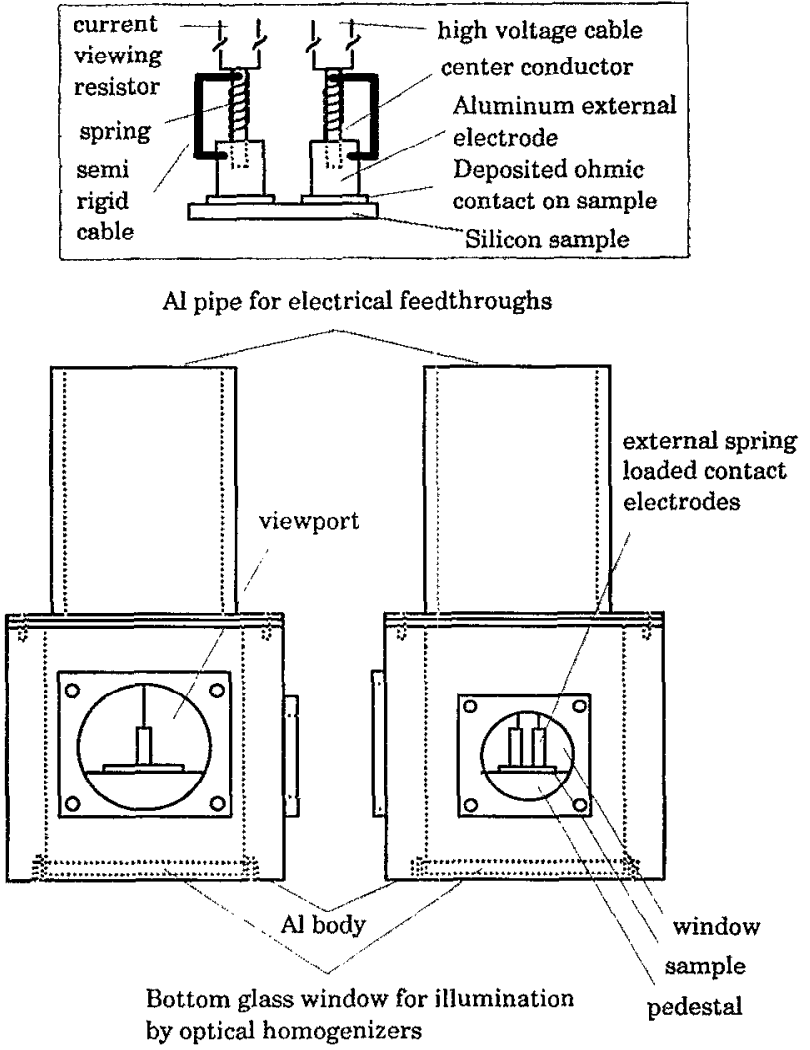

Side View

Front View

FIG. 2. Sketch of the Kerr cell with details of the contact electrodes shown in the inset.

nation through optical homogenizers in future. The electrical feedthroughs are brought inside the cell by coaxial cables from the top through an aluminum pipe which is closed at the bottom. The coaxial feedthroughs terminate on specially designed spring loaded contact electrodes which are rectangular in shape and create a uniform field geometry. The external contact electrodes are $9 \mathrm{~mm}$ in length, $5 \mathrm{~mm}$ thick, $15 \mathrm{~mm}$ high, and are normally spaced about $3 \mathrm{~mm}$ apart. The electrode length and spacing is chosen to prevent any contribution from the electrodes to the induced birefringence. The electrode spacing can, however, be changed by bending the semirigid coaxial cable to which the electrodes are attached. The entire cell assembly has been designed with Teflon $O$-ring seals to prevent any leakages. The Kerr cell is located in a vent hood and rests on a specially constructed lab jack which can be raised or lowered accurately. The cell is filled with laboratory grade high-purity nitrobenzene ( $99 \%$ pure) which serves as the electro-optic medium. The nitrobenzene purity level is not critical if it is used for pulsed measurements; however, for dc measurements special purification techniques will be required. Such purification methods include treatment with a weak basic solution to remove traces of acid, treatment with a drying agent to remove water, fractional distillation and fractional freezing. ${ }^{9}$ Nitrobenzene is chosen as the electro-optic medium because it has the highest reported Kerr coefficient, it has high transmissivity for $\mathrm{He}-\mathrm{Ne}$ wavelength $(632.8 \mathrm{~nm})$ and has a high breakdown strength.
The response time of the nitrobenzene Kerr cell for an applied electric field is about a nanosecond and serves as the upper bound for the temporal resolution of the system. Thus this measurement apparatus is ideally suited for transient field measurements requiring nanosecond accuracy,

As can be observed from the analytical expression given earlier, knowledge of the $s_{11}$ coefficient of nitrobenzene is essential for calculating the surface electric fields. The $s_{11}$ value is determined using the same experimental setup but the sample is removed from the Kerr cell and the external electrodes are brought closer to generate the high fields necessary to cause sufficient phase retardation. As the applied high voltage can be accurately measured and the external electrodes are rectangular in shape, a uniform field geometry is created between the electrodes whose value can be simply determined by dividing the applied voltage by the electrode separation. The value of $\Delta_{E}$ is experimentally determined using Eq. (2) and this value is substituted in Eq. (3) to determine the value of $s_{11}$. This procedure is repeated at different voltage levels and the average value of $s_{11}$ is found out to be $-7.6923 \times 10^{-15}$ $\mathrm{cm}^{2} / \mathrm{V}^{2}$.

\section{EXPERIMENTAL RESULTS}

Experiments were conducted using high-resistivity (12 $000 \Omega / \mathrm{cm}$ ) silicon substrates with aluminum contacts deposited on them. The contacts were rectangular in shape having dimensions of $0.5 \mathrm{~mm}$ (gap length), $2 \mathrm{~cm}$ (width), and $5000 \AA$ (thickness) and the contacts were unalloyed. The contact edge perturbations were negligible and the contacts were measured to be ohmic in nature. Figures 3 show the plot of the measured surface electric field between the contact spatial points for various time instants for different applied voltages. These measurements were done by measuring the fringe bending at various spatial points and for different time instants. The details of the measurement method are described in Refs. 4 and 6. This sample withstood an applied voltage of $2206 \mathrm{~V}$ which for a $0.5 \mathrm{~mm}$ gap corresponds to an average field of about $44.12 \mathrm{kV} / \mathrm{cm}$. The switch underwent surface flashover at a voltage of $2319 \mathrm{~V}$ as was observed by a sudden fall in the voltage across the switch accompanied by a sharp rise in current on the oscilloscope. The surface electric field during flashover could not be plotted as multiple fringe movement occurred which made it difficult to decipher the data. Thus the flashover threshold for this sample was between 44.12 and 46.38 $\mathrm{kV} / \mathrm{cm}$. For the graphs shown, the spatial points which gave the most pronounced fringe shifts were chosen as the representative points and they were then plotted and joince by a smooth curve. As can be observed from Fig. 3(a), the electric field is nonuniform in space but uniform in time for lower applied voltage. As the applied voltage is increased [Fig. 3(b)] the electric field starts becoming nonuniform in time (duration of the voltage pulse). As the voltage is increased further, the nonuniformity in time is more pronounced. These results compare favorably with the results obtained by Donaldson, ${ }^{3}$ who had observed asymmetric spatial electric field distribution between the contacts of a silicon switch. 


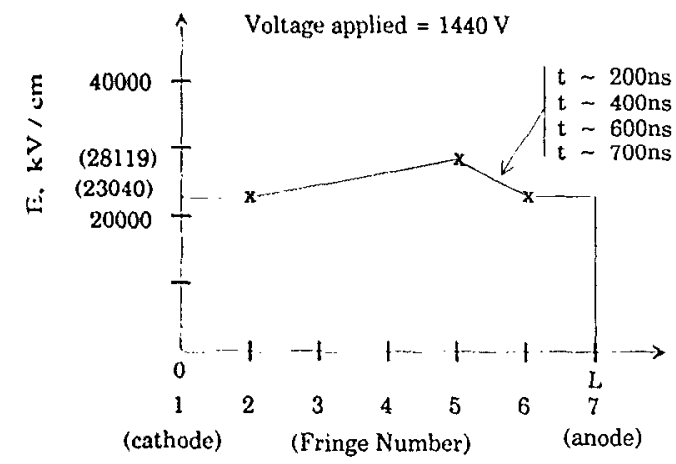

(a)

Contact separation, number of fringes

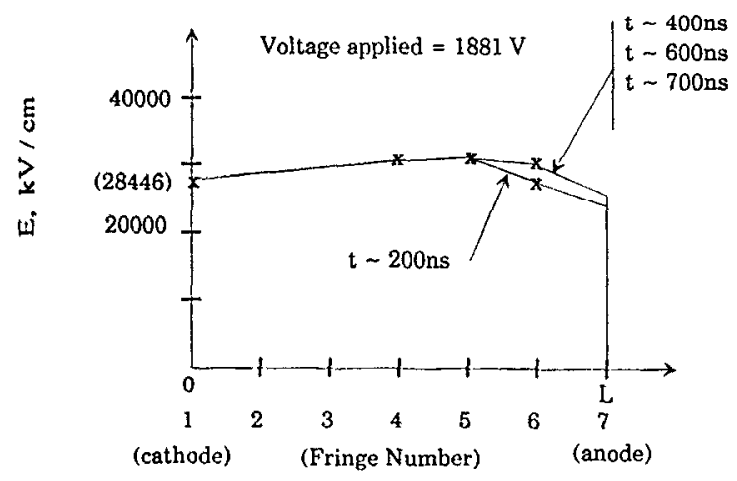

Contact separation, number of fringes

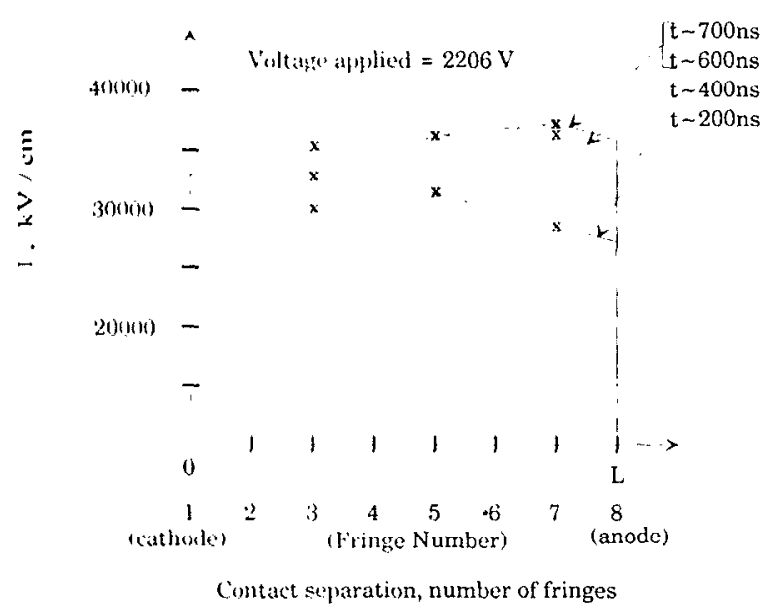

FIG. 3. Plot of surface fields against space for various time instants and applied voltages: (a) $1440 \mathrm{~V}$, (b) $1881 \mathrm{~V}$, (c) $2206 \mathrm{~V}$.

The total error in the absolute measurement of surface electric fields using this technique is about $30 \%$, which can be attributed to the following factors. The error in the measurement of the $s_{11}$ coefficient is the maximum (about $25 \%$ ) which can be substantially reduced by specially designing a Kerr cell suited for calibration purposes only. The error in determining the length (of the ohmic contacts) is typically $3 \%$ which is due to the presence of fringing fields. The error in determining the fringe shifts is about 4\% which again depends upon the method used for calculating the fringe shifts and the amount of fringe shifts obtained. This leads to a total worst case error of about
$30 \%$ which can be substantially reduced by making more accurate measurements of $s_{11}$. The $25 \%$ error in the measurement of $s_{11}$ was due to error in the measurement of the length of the external electrodes $(4.5 \%)$, error in the measurement of the electrode separation (4.5\%), error in the measurement of the fringe shifts $(7 \%)$, and error in the measurement of the applied voltage (1\%). This error can be reduced by building a Kerr cell with precisely machined, long, fixed electrodes. In that case, the length and electrode separation would be fixed and would contribute negligibly to the error. Further, by having longer electrodes, more fringe shifts can be obtained for the same applied voltage and it would reduce the error. Superior data acquisition systems which can directly digitize the fringe image would also be advantageous in the reduction of the error. In our case, the purpose of the external electrodes was to make a good electrical contact with the contacts on the sample and they were therefore not very precisely machined. Further, as the samples were of different dimensions and were changed periodically, the external electrode separation had to be changed to match the sample. This contributed to the error in determining the electrode separation during calibration. In addition, the external electrodes were designed to contribute negligibly to the sample electric field, and they were much smaller in length than the contact length. Thus during calibration (when the sample was removed), only partial fringe shifts were obtained, which increased the error in determining the fringe shifts.

It should be, however, noted that the relative error associated in the measurement of the electric field at adjacent spatial or temporal points is much less than $30 \%$. Thus the shape of the electric field distribution in time or space is quite accurate, the limitations being imposed only on the ability to distinguish between the fringe shifts at the points of interest and the error in length due to the fringing fields. As this error is about $7 \%$, we can say that the relative error of this measurement technique is about $7 \%$, as a different value of $s_{11}$ will act as a scaling factor but not change the overall distribution of the surface electric field. The relative measurements are very important as we are more often interested in knowing the change in surface fields with time and space (as this can help locate problem areas) than the actual absolute values.

\section{v. DISCUSSION}

A new electro-optic technique for the measurement of surface electric fields was developed and the surface fields were determined with a temporal resolution of $100 \mathrm{~ns}$ and a spatial resolution of $50 \mu \mathrm{m}$. The measurement technique required the knowledge of the electro-optic coefficient of nitrobenzene $\left(s_{11}\right)$ which was experimentally determined. The flashover threshold of silicon photoconductive power switches in nitrobenzene was found out to be between 40 $50 \mathrm{kV} / \mathrm{cm}$ by performing measurements on a number of samples having unalloyed aluminum contacts. The surface 
electric fields were observed to be nonuniform in time and space. The temporal nonuniformity was observed at higher applied voltages. This indicates that the semiconductor surface has an important role to play in determining the surface fields and on the flashover threshold. Evidence of residual surface charge was observed at higher voltages by the persistence of fringe shifts (electric field) after the voltage pulse duration. The spatial and temporal distribution of the surface electric fields on simple planar silicon samples has thus been determined using this measurement technique.

\section{ACKNOWLEDGMENTS}

We wish to acknowledge Dr. Robin Chen for fabricating some of the semiconductor samples, Dr. Bob Druce of Lawrence Livermore National Laboratory for the loan of the streak camera, and Dr. James Thompson of the Uni- versity of New Mexico for many useful discussions. This work was supported by AFOSR under AFOSR contract No. 89-0253.

'D. C. Wunsch and A. Erteza, Rev. Sci. Instrum. 35, 816 (1964).

${ }^{2}$ J. E. Thompson, M. Kristiansen, and M. O. Hagler, IEEE Trans. Instrum. Meas. 25, 1 (1976).

${ }^{3}$ W. R. Donaldson, SPIE Paper No. 87214 (1987).

${ }^{4}$ K. H. Schoenbach, J. S. Kenney, A. Koenig, B. J. Ocampo, R. F. K. Germer, and H. J. Schulz, in Proceedings of the Eighth IEEE Pulsed Power Conference, edited by R. White and K. Prestwich (San Diego, CA, 1991), pp. 105-108.

${ }^{5}$ H. P. Sardesai, W. C. Nunnally, and P. F. Williams, in Proceedings of the SPIE Conference on Optically Activated Switching, edited by F. J. Zutavern (Boston, MA, 1990), pp. 237-248.

${ }^{6}$ H. P. Sardesai, W. C. Nunnally, and P. F. Williams, in Proceedings of the Eighth IEEE Pulsed Power Conference, edited by $\mathrm{R}$. White and $\mathrm{K}$. Prestwich (San Diego, CA, 1991), pp. 1028-1031.

${ }^{7}$ H. P. Sardesai, M.S. thesis, University of Texas at Arlington, 1991.

${ }^{8}$ E. F. Kingsbury, Rev. Sci. Instrum. 1, 23 (1930).

${ }^{q}$ H. J. White, Rev. Sci. Instrum, 6, 22 (1935). 